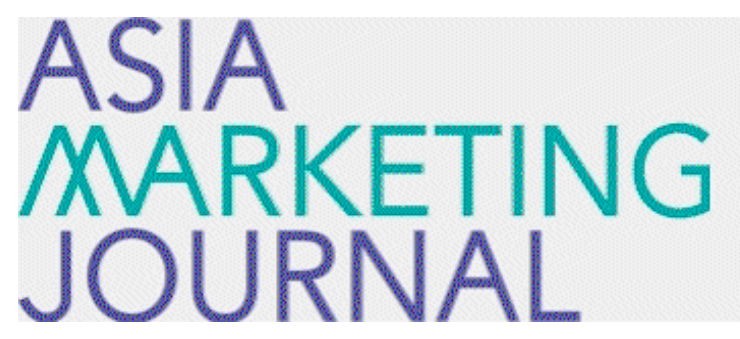

ASIA MARKETING JOURNAL

Volume 11 | Issue 4

Article 4

$1-30-2010$

\title{
컬러 불일치가 브랜드 태도에 미치는 영향
}

\section{Sang Eun Lee}

Sang Yong Kim

Follow this and additional works at: https://amj.kma.re.kr/journal

Part of the Marketing Commons

\section{Recommended Citation}

Lee, Sang Eun and Kim, Sang Yong (2010) "컬러 불일치가 브랜드 태도에 미치는 영향," Asia Marketing Journal: Vol. 11 : Iss. 4 , Article 4.

Available at: https://doi.org/10.53728/2765-6500.1245

This Article is brought to you for free and open access by Asia Marketing Journal. It has been accepted for inclusion in Asia Marketing Journal by an authorized editor of Asia Marketing Journal. 


\title{
컬러 불일치가 브랜드 태도에 미치는 영향: \\ 자아이미지 일치성의 조절효과를 고려하여
}

\section{The Effect of Color Incongruity on Brand Attitude: Moderating Effect of Self-Image Congruence}

\author{
이 상 은(Lee, Sang Eun)* \\ 김 상 용(Kim, Sang Yong)**
}

본 연구는 통합적 브랜드 관리에 있어 매체 간 컬러의 불일치가 브랜드 태도에 긍정적인 영향을 미치며, 브랜드-소비자 자아 일치성이 이 영향에 조절적 효과를 가져 온다는 가정을 실험을 통해 증 명하였다. 매체는 컬러의 영향을 살펴보기 위하여 시각적 영향이 부각되는 매체로 제한하였으며, 컬 러 이외의 시각적 요인은 일관되도록 하였고, 브랜드에 대한 친숙도가 낮은 브랜드, 브랜드에 대한 이전의 지식이 없는 브랜드를 대상으로 하였다.

결과는 매체 간 컬러의 불일치에 따른 브랜드 태도는 컬러의 일관성에 따른 브랜드 태도에 비해 높게 나타났으며, 브랜드-소비자의 자아일치가 낮은 경우에 높은 경우보다 태도의 변화가 높게 나타 나 가설이 모두 지지되었다. 이 결과는 브랜드의 관리적 요소로서 브랜드 표현, 즉 시각적 요인 중 컬러를 통하여 브랜드에 대한 인식을 높이고, 긍정적인 브랜드 태도를 형성할 수 있다는 것을 제안하 였다는 것에 의의가 있다. 또한 본 연구는 브랜드 관리자의 직관에 의지하던 브랜드 표현의 일관성과 다양성의 수준이 일반적 의식보다 더 폭넓다는 것을 실증적으로 증명하고, 이전의 연구에서 성숙브랜 드(mature brand)를 리포지셔닝(repositioning)하는 전략으로만 연구되었던 불일치를 신생 브랜드의 인지를 높이기 위한 전략으로 그 범위를 확장시켰다고 할 수 있다.

핵심개념: 컬러, 불일치, 브랜드 태도, 소비자 자아일치성

* 홍익대학교 시각디자인전공 박사과정, 우송대학교 강사, 강원대학교 강사(bytaiji @hotmail.com)

** 고려대학교 경영대학 교수(sangkim@korea.ac.kr), 교신저자 


\section{I. 연구배경 및 목적}

소비자들은 너무 많은 양의 정보에 노출되어 있다. 브랜드는 수많은 양의 정보들 속에서 브 랜드를 다른 정보들 보다 효과적으로 인식시켜 야 하는 과제에 당면해 있는 것이다. 이 때문에 이전의 많은 연구자들은 브랜드 커뮤니케이션 의 통합적 관리에 있어 소비자에게 브랜드를 명확하게 인식시키기 위해 브랜드의 메시지는 일관성을 유지하여야 한다고 주장해왔다. 반면, '불일치(Incongruity)'를 주장하는 연구자들은 이미 익숙하거나 새롭지 않은 자극에 소비자들 은 반응하지 않으며, 의외성이 브랜드를 인식시 키고 환기시키는 데 효과적이라고 주장한다. 본 연구는 매체 간 커뮤니케이션의 불일치가 소비 자의 인식에 영향을 미칠 수 있다면, 이것이 브 랜드 태도에 긍정적인 영향을 가져올 수 있을 것이라는 주장을 중심으로 하고 있으며, 효과적 인 불일치의 요소로서 시각적 요인을 제시하고 자 한다.

브랜드의 커뮤니케이션 요소간의 불일치에 대 한 이전의 연구들은 브랜드 친숙도가 형성된 브랜드의 경우, 브랜드 태도를 향상시킨다는 것 을 증명하였으나(Machleit et al. 1993; Sjödin and Törn 2006), 대부분이 시각적 요인과 언어 적 요인의 불일치(Heckler and Childers 1992), 혹은 이미 형성된 브랜드의 스키마(schema)와 자극의 불일치(제품 카테고리의 연상과 제품의 외형의 불일치, 기대와 광고의 불일치)에 대한 내용뿐이며, 브랜드의 통합적 관리 차원에서 여 러 매체의 관리, 특히 시각적 요인을 통한 관리 에 대한 연구는 부재하다.
이에 본 연구는 한 브랜드의 각 매체 간 컬러 의 불일치는 소비자의 브랜드 태도에 긍정적 영향을 미칠 것이라 가정하고 시각적인 영향이 큰 매체를 중심으로 살펴보고자 한다. 본 연구 의 결과는 커뮤니케이션 요인의 불일치, 혹은 의외성이 소비자의 인식에 자극을 주며, 브랜드 에 대한 인식과 태도를 긍정적으로 만들 수 있 는 새로운 전략을 제안하게 될 것이다.

\section{II. 이론적 배경}

\section{1 브랜드의 시각적 아이덴티티로서의 컬러}

브랜드 아이덴티티(brand identity)는 기업이 창조하고 유지하기 위해 불러일으키는 브랜드와 관련된 일련의 독특한 연상이다(Aaker 1996). 이는 소비자 입장에서 연상된 이미지를 뜻하는 브랜드 이미지와는 구별되며, 기업의 입장에서 소비자가 자사의 브랜드를 보고 떠올렸으면 하 는 연상을 의미한다. 브랜드 아이덴티티는 소비 자들과 브랜드가 접촉하는 여러 가지 요소에 의해 형성되는데(최원주, 김흥규 2005), 브랜드 의 각 요소들은 소비자에게 브랜드에 대한 인 식과 친밀성을 증대 시키고, 구매 가능성을 높일 수 있는 수단이 된다(Shimp 1999; Keller 1998). 인간의 뇌에 전달되는 모든 자극의 3 분의 2 는 시각적이라는 Zaltman(1996)의 주장처럼 브랜드 아이덴티티의 형성에 있어 시각적 요소는 중요 한 부분을 차지하고 있다. 또한 시각적 요소는 언어적 정보에 더해졌을 때, 기억을 향상시키는 
데에 효과가 있다(Tavassoli 1998; Schmitt and Simonson 1997). 그 중에서도 컬러는 시각적인 것들 중 소비자가 사물(제품, 패키지, 매장 디스 플레이)을 인식하고 판단하기 위해서 지각하고 통합해야 하는 여러 시각적 요소(형태, 크기)중 하나이다(Triesman 1991). 컬러는 브랜드의 본 질적인 브랜드의 의미를 투영하는 유일한 구성 요소(Bottomley and Doyle, 2006)라고 할 수 있 으며, 컬러경험은 브랜드에 의미를 부여하거나, 존재하는 의미와 상징적 연상을 강화하거나 높 이는 특징을 가지고 있다(Garber and Hyatt 2003).

이전의 브랜드 커뮤니케이션과 관련된 컬러 연구들은 잡지광고에서 특정 컬러의 사용 (Schindler 1986; Lee and Barnes 1990), 흑백 광고와 컬러 광고의 비교(Sparkman and Austin 1980; Meyers -Levy and Peracchio 1995), 특 정한 컬러에 대한 소비자 반응(Bellizzi et al. 1983; Gorn et al. 1997)과 같은 분야에서 이루 어져 왔다. 그러나, 브랜드는 여러 커뮤니케이션 경로를 통해 고객에게 인식되기 때문에 여러 매체를 함께 관리하는 것은 매우 중요하다고 할 수 있음에도 불구하고, 브랜드 아이덴티티를 형성하는 여러 매체 간의 컬러 조절에 대한 연 구는 부재하다.

\section{2 브랜드 일관성과 불일치}

\subsection{1 브랜드의 일관성}

브랜드 커뮤니케이션의 일관성을 주장하는 여 러 문헌들은 고객과 다른 이해집단이 브랜드를 빠르고 정확하게 인식하도록 하기 위해서는 브
랜드를 동일화하는 마크와 로고와 같은 시각적 요인의 일관성을 유지하는 것이 경쟁우위를 선 점하고(Duncan and Moriarty 1997; Keller 1998; 최원주, 김흥규 2005), 목표 고객에게 효과적으 로 도달할 수 있는 확률을 높일 수 있는 방법 (Schmitt and Simonson 1997)이라고 주장한다. 마케팅 커뮤니케이션에서 커뮤니케이션의 소재 의 면모는 일관되어야 한다고 주장하는 Moore 와 Thorson(1996)의 “단일면모” 접근법(The mandated "One Look" approach)과 자극에 의한 선호는 '단일한 노출'을 통해 증가한다는 Zajonc(1968)의 친숙효과(Familiarity Effect)이 론들은 이 같은 주장을 뒷받침해주며, 그 외에 도 많은 연구들에서 브랜드 컨셉의 일관성에 대해 강조하고 있다(Park et al. 1986; Keller 1993; Broniarczyk and Alba 1994; Leigh 1992; Scott and Batra 2003). 그러나 이러한 일관성 유지 관점은 모든 형태의 커뮤니케이션이 공통 적 면모를 가지고 있다고 하더라도 전략적 측 면이 결합되지 않으면, 그 효과를 충분히 발휘 못 할 가능성이 있다는 결점을 가지고 있다.

Bornstein(1989)은 친숙효과(Familiarity effect) 의 한계에 대해, 지루함 효과(Boredom effect) 와 같이 노출의 반복과 연장은 개인을 자극에 대해 더 안정되게 하며 너무 많은 노출은 자극 에 대해 지루하게 한다고 하였다. 일관성을 유 지하는 통합적 관리는 단일한 전략으로는 완벽 할 수 없으며, 각 커뮤니케이션 도구들과 함께 시너지 효과를 가져올 수 있도록 상호보완 되 어야 하는 것이다(최원주, 김흥규 2005). 


\subsection{2 브랜드의 불일치: 적절한 불일치}

(Moderate Incongruity)

불일치에 대한 연구들은 인식, 사회심리, 소비 자행동, 광고이론 등의 연구들, 즉 자극에 대한 생리학적인 인지, 소비자가 어떻게 마케팅 정 보들을 처리하는가, 정보들은 기억 속 스키마 (Schema)들과 어떻게 비교되는가, 스키마에 있 는 명확한 요소들의 관련성은 정보의 기억을 어 떻게 촉진하는가와 연결되어있으면서, 그보다 확 장된 내용들을 제시한다. 이 같은 연구들에서 언 급한 불일치에 대한 이론들 중 스키마 이론 (Schema theory), 정보처리 접근법(Information Processing Approach), 불일치 현저성(IncongruitySalience)이론들은 불일치성과 관련성의 효과를 고려하는데, 지각자(perceiver)는 자신의 스키마 (Schema)와 정황적으로 불일치하는 대상에 대해 주의를 기울인다는 것이다. Guido (2001)에 따르 면, 불일치 현저성(Incongruity- Salience)은 자 극이 일어나는 상황에 따라, 그 배경(context) 이 어떤지에 따라서 달라지는데, 상황적인 새로 움(contextual novelty)과 다른 물리적 성질(위
치, 대비, 크기)이 함께 작용해야 소비자의 주의 (attention), 선호(prefer), 흥미를 가져올 수 있 다고 하였다. 불일치 현저성은〈표 1〉에서 보듯 이 외부적 자극으로 시작되는 상향정보처리이 므로, 소비자 입장에서 이 자극은 마케팅 커뮤 니케이션 요소에 의한 자극을 가져온다고 볼 수 있을 것이다. 관련 현저성(relevant-salience) 에 비해 본 연구에서 다루고 있는 브랜드에 대 한 지식(혹은 schema)이 형성되어있지 않은 브 랜드에 적합할 것으로 보인다.

이외에도 불일치에 대한 이론으로 인지적 이 론 중 신기효과(Novelty Effect)가 있는데, 이 이론은 지각자(perceiver)가 새로운 자극에 대해 더 즐겁고 흥미롭다고 느낀다(Berlyne 1971)는 것이다. 이에 관한 연구들에서는 새로움(novelty) 은 전체적 인식 기억에 영향(Fabiani and Donchin 1995; Kishiyama and Yonelinas 2003)을 가져 오며, 선호와 양의 관계를 가지고 있다고 주장 한다(Bruce and Green 1990). 그리고 컬러, 크 기, 명암, 의미적 범주와 같은 자극적 차원의 변 수 항목들이 새로울(novel) 때에(Wallace 1965) 인 식적 기억이 향상된다고 하였다. 특히 Kishiyama

〈표 1〉A Dichotic Theory of salience (Guido 2001)

\begin{tabular}{c|cc}
\hline salience type & Incongruity-salience & Relavant-salience \\
\hline 자극 유형 & 지각 & 기억 \\
인식 과정 유형 & 상향정보처리* & 하향정보처리** \\
탐색행동 유형 & 탐구적, 입문적 탐색행동 & 목표지향적 탐색행동 \\
학습유형 & 우발적 학습 & 의도적 학습 \\
발생 이유 & 불일치(자극의 내용이나 중요도) & 몰입, 관계(개인의 목표나 지식) \\
주의(attention)유형 & 무의식적인 주의(수동적 주의) & 자발적 주의(계획적 주의) \\
\hline
\end{tabular}

주) * 상향정보처리: 들어오는 자료를 있는 그대로 분석하여 의미를 파악. 기존의 지식이 크게 작용하지 않는다.

** 하향정보처리: 들어오는 자료를 있는 그대로 분석하여 의미를 파악하지 않고, 머리(기억)속에 저장되어 있는 지식을 끌어내어 그 지식이 제공하는 틀을 이용하여 정보를 처리한다. 
와 Yonelinas(2003)의 연구에서는 컬러를 조절 하여 신기효과(novelty effect)를 측정, 회상과 기억에 효과가 있다는 것을 밝혀냈다.

불일치와 브랜드에 대한 실증적, 이론적 선행 연 구들을 살펴보면, 브랜드 친숙도(brand familiarity) 가 형성된 브랜드의 경우, 불일치의 고의적 사 용은 성숙기의 브랜드(mature brand)에 대한 흥 미를 되살릴 수 있는 이점이 있으며(Machleit et al. 1993), 브랜드와 그 브랜드의 특정한 커 뮤니케이션 일부의 불일치는 브랜드에 대한 소 비자의 주의, 동화(elaboration), 혼란스러운 단 서들을 해석하려는 노력, 감정적 반응, 회상 (Sjödin and Törn 2006) 등을 증가시킨다는 주 장을 제시하였다. Lange와 Dahlen(2003)은 브 랜드와 광고의 불일치는 브랜드 태도와 브랜드 기억은 증가하는 반면, 브랜드 친숙도(brand familiarity)가 낮은 브랜드에서 브랜드와 광고 의 불일치는 브랜드 기억을 감소시킨다는 결과 를 보여주었다. 이 외에도 광고의 언어적, 비언 어적 요인의 불일치(Heckler and Childers 1992), 제품 카테고리의 연상과 제품의 외형의 불일치 (Campbell and Goodstein 2001; Meyers-Levy and Tybout 1989; Peracchio and Tybout 1996), 기대와 광고의 불일치(McQuarrie and Mick 1999)에 대한 연구들이 진행되어왔다.

그러나 이와 같은 불일치에 대한 논증들은 불 일치가 소비자의 인지, 태도에 미치는 긍정적 영향들에 대한 것이다. 그러나 그 결과는 불일 치의 정도에 따라 현저히 달라질 수 있다.

Schmitt와 Simonson(1997)은 브랜드 아이덴 티티 관리의 불일치(다양성)에 대해 아이덴티티 가 불명확해 질 수 있는 가능성이 있다고 하였 으며, Light(1994)는 통합이 아닌 불일치는 불확
실성을 가져올 수 있다는 점을 지적하였다.

Tavassoli(1998)의 부호화 다양성 이론(Encoding variability theory)은 소비자는 다양한 배경 (context)에서 같은 메시지를 받을 때 그 메시 지는 기억 속에 암호화 되며, 머리 속에서 더 강하고, 명확하고, 더 접근하기 쉬운 정보가 형성됨으로써, 연상될 가능성을 높여줄 것이 라고 하였다. 이와 비슷한 이론으로는 사회 판 단 이론(Social Judgment Theory)과 동화효과 (assimilation effect)가 있다. 사회 판단 이론 (Sheriff and Hovland 1961)은 태도의 표현에 대한 것으로서, 개인은 입력된 메시지와 비교할 기준(frame of reference)의 역할을 하는 이전 의 태도를 가지고 있다는 이론이다. 이 이론에 따르면 입력된 메시지는 개인의 기준에 따라 수락범위(latitude of acceptance)와 거절범위 (latitude of rejection)로 나뉘어 판단된다(Pimentel and Helker 2003). 동화효과(Assimilation Effect) 는 제시물이 지각자(perceiver)의 수락범위에 들게 되면 동화효과가 일어나게 되는데, 이는 제시물이 실제로 그런 것 보다 더 유사하게 인 지한다는 것이다. 반대로, 거절범위에 들게 되면 대조효과(Contrast Effect)가 일어난다(Scott and Batra 2003). 결과적으로 불일치는 소비자가 인 지할 때, 수락범위 안에 있어야 긍정적 태도를 불러올 수 있는 것이다.

Pimentel과 Helker(2003)는 연구에서 수락범 위(adaptation level) 안에 있는 로고와 벗어난 로고 디자인을 보여준 결과 수락 범위를 벗어난 로고의 모든 수준(level)의 디자인에 대한 선호 (preference)가 떨어진 것을 실증하였고, Garber 와 Hyatt(2003)는 제품 포장의 컬러 변화에 대 한 소비자의 반응에 대한 연구에서 소비자는 
제품과 포장에서 혁신적인 시각 요인에 호감을 가진다고 주장하면서, 단 그 시각적 의미가 제 품 카테고리와 일관성이 있어야 한다고 하였다. 개인은 이미지의 의미가 분명하지 않은 시각이 미지가 변화되는 것을 좋아하지 않는다(Pimentel and Helker 2003)는 것이다. 또한 불일치에 대 한 스키마 이론(Schema theory)에 대한 연구들 에서도 소비자들은 중간 수준의 불일치를 선호 한다고 주장한다(Mandler 1982; Meyers-Levy and Tybout 1989).

적절한 불일치(Moderate Incongruity)란 각각 의 브랜드 요소들이 브랜드 아이덴티티를 형성 하는데 있어 각기 수행하는 역할이 다르다는 것 (Keller 1998)을 이해해야 하고, 그 효과를 극대 화하기 위해서는 일방적인 일관성 보다는 중심 적 메시지를 일관성 있게 유지하면서 각각의 매체의 특성에 따라 관리를 해야 한다(Sirgy 1997)는 것을 의미한다. 브랜드 아이덴티티의 시각적 요소, 즉 브랜드 표현을 관리하는데 있 어 일관성 혹은 불일치성을 선택하는 것은 불 일치의 적절한 정도를 유지할 수 있다면, 다양 성을 유지하면서도, 일관된 메시지를 전달하고 이를 통해 긍정적인 소비자의 브랜드 태도를 가져올 수 있을 것이다.

본 연구는 메시지의 일관성을 유지하기 위해 로고, 제품의 이미지(picture), 카피, 레이아웃 (lay-out)을 일관성 있게 표현하고, 브랜드 커뮤 니케이션 통로인 각 매체 간 사용된 컬러를 불 일치시킴으로써 적절한 불일치의 정도를 유지 할 수 있을 것이라고 주장하며, 각 매체 간의 컬러의 불일치는 친숙도가 낮은 신생브랜드의 경우에 소비자의 브랜드에 대한 태도에 긍정적 인 영향을 가져올 것이라고 가정한다.
가설 1: 브랜드 친숙도가 낮을 때, 매체 간 컬 러의 불일치는 브랜드에 대한 소비 자의 태도에 긍정적인 영향을 줄 것 이다. 즉, 브랜드 친숙도가 낮을 때, 불일치 정도가 클수록, 소비자의 브 랜드 태도는 더 긍정적일 것이다.

\section{3 브랜드-소비자 자아와의 일치}

Guido(2001)는 불일치 자극은 지각자에 따라 그 결과가 달라진다고 하였다. 주의를 끄는 자 극은 모든 개인에게 동일하지 않으며, 개인의 본성과 배경(context)이 결합하여 같이 작용해 야 하는 것이다. 브랜드 전략은 그 메시지를 받 는 대상을 고려하여야 할 것이다.

브랜드와 소비자 자아와의 관계는 소비자가 자신의 이미지를 다른 사람에게 전달하는데 있 어 브랜드의 상징적 이미지를 이용(Aaker 1996) 하는 성향 때문에 그 개념 자체가 소비자의 특 성을 대표한다고 볼 수 있다. 자아(Self-image) 는 개인의 신체적 상태(physical being) 뿐만 아니라 개인 자신에 대한 정의(definition)와 평 가도 포함하는 개념으로서, 제품과 서비스의 구매를 포함하는 인간의 모든 행동을 반영한다 (Birdwell 1968). 소비자는 브랜드에 대해 실제 적 자기 일치가 높을 때, 브랜드에 대한 긍정적 인 태도를 보이며, 실제적 자기 일치가 낮을 때 에는 브랜드에 대한 부정적인 태도를 보이는 경향(Moore and Thorson 1996)을 보인다. 이는 소비자들에게 자아와 개인적 정체성(personal identity)을 강화하는 제품이나 브랜드를 구매하 도록 하는 동기가 유발되기 때문이다(Sirgy and Johar 1991). 
여러 연구에서 Self-image congruence, selfcongruity, image congruence, self-concept congruity 등의 용어로 쓰이고 있는 자아 일치 는 소비자의 자아(실제적 자아, 이상적 자아)와 제품, 브랜드, 매장 등의 사용자 이미지(혹은 개 성)의 일치(Kressmann et al. 2006)로 정의될 수 있다. 자아 일치는 제품의 선호, 브랜드선호, 브랜드 선택, 소비자 만족, 매장 충성도를 예상 할 수 있는 요인으로 언급되고 있다.

브랜드와 소비자의 관계의 자아 일치성을 연 구한 연구들에서 자아 일치성은 브랜드의 구매 의도(Sirgy 1985)와 브랜드 충성도에 긍정적 영향(Malhotra 1988; Sirgy and Samli 1985) 을 가져오며, 소비자와 브랜드의 관계를 강화 (Fournier 1998; Aaker 1996; Thorbjrnsen et al. 2002; Aaker et al. 2004; Bhattacharya and Sen 2003)하고, 소비자가 인식한 브랜드의 개 성과 소비자 자아가 일치하는 브랜드를 선택 (Birdwell 1968)하는 경향이 있다는 것을 증명 하였다. 특히 Dolich(1969)와 Graeff(1996)는 소 비자의 자아와 브랜드 이미지가 일치 할수록 브랜드에 대한 선호 및 긍정적 태도가 형성된 다고 주장하였다.

Guido(2001)가 제시한 불일치 이론(〈표 1〉참 고)에서 불일치는 필요에 의한 정보의 탐색이 아닌 우발적으로 이루어지며, 미리 형성되어있 는 스키마(schema)가 없는 상태에서 자극의 불 일치에 의해 이루어진다고 하였다. 또한, Kirmani 와 Shiv(1998)의 연구에서는 문제관련동화 정도 (Issue-relavant elaboration level)가 높을 때, 소스 일관성(source congruity)은 브랜드 태도 에 긍정적이며, 반대로 문제관련동화 정도가 낮 을 때, 소스 일관성이 브랜드 태도에 미치는 영
향이 작으며, 브랜드 태도를 형성하는데 주변적 단서나, 발견적 방법을 사용한다는 실증적 결과 를 제시하였다.

이는 브랜드의 매체 간 불일치하는 브랜드 표 현 요소들에 대해 태도가 좋아지는 정도가 소 비자의 자아 일치성이 낮은 실험대상에게 더 크게 나타날 수 있다는 가능성을 제시한다. 즉, 신생브랜드(브랜드 친숙도가 낮은)가 타겟으로 설정한 소비자 뿐 아니라 그 외의 소비자들에 게도 브랜드에 대한 인식과 태도를 긍정적으로 만들 수 있는 가능성을 의미하는 것이다.

본 연구에서는 매체 간의 불일치를 소비자의 자아 일치성이 낮은 그룹, 즉 높은 그룹에 비해 브랜드에 관심이 낮은 소비자들의 브랜드 태도 를 높일 수 있는 효율적 방법으로 제시하며, 다 음과 같은 가설을 도출하였다.

가설 2: 브랜드 친숙도가 낮을 때, 매체 간 컬 러의 불일치 정도가 브랜드에 대한 소비자의 태도에 긍정적인 영향을 주는 정도는 브랜드-소비자 자아 일 치성이 낮을 때가 높을 때보다 더 클 것이다. 즉, 브랜드 친숙도가 낮 을 때, 브랜드-소비자 자아 일치성 이 낮을수록, 불일치 정도가 소비자 의 브랜드 태도에 주는 긍정적인 영 향은 더 커질 것이다.

\section{4 브랜드 태도}

브랜드의 여러 도구들 간의 불일치에 대한 연 구들에서는 광고의 요소들이 불일치할 때, 광고 와 여러 요소에 대한 기억이 향상(Heckler and 
Childers 1992)되며, 광고와 브랜드에 대한 태도 가 강화(Lee and Mason 1999; Lee 2000)되고, 호기심과 흥미(Muehling and Laczniak 1988), 메시지에 대한 몰입(involvement), 신뢰의 증가 (Lee 2000)를 가져온다고 하였다. 불일치가 가 지는 이러한 영향들은 브랜드에 대해 인식적이 고, 조심스런 동화를 가져오며(Kent and Allen 1994) 더 호의적인 태도(Hecker and Childers 1992; Lee 2000)를 가져올 것이다.

브랜드에 대한 소비자들의 전반적인 평가를 기초로 정의되는 브랜드 태도는 브랜드 선택과 같은 소비자의 행동에 기초가 되는 개념(Keller 1993)이며, 가장 높은 수준의 가장 강력한 브랜 드 연상 유형(Keller 1998)으로 제품과 관련 없 는 속성과 자아를 표현하는 도구로서의 이점을 (Rossiter and Percy 1987) 가지고 있다. 또한 브랜드에 대한 연상들은 기본적인 속성에 대한 정보보다 기억 속에 더 오래 남고 또 기억하기 가 더 쉬우며(Chattopadhyay and Alba 1988), 브랜드에 노출되자마자 자연스럽게 활성화될 가능성이 더 높고 바로 그 뒤에 행해지는 브랜 드 선택을 유도(Berger and Mitchell 1989)하게 되는 특성을 가지고 있다.

이러한 브랜드 태도의 속성들은 브랜드를 처 음 접하는 시점, 즉 본 연구에서 설정한 브랜드 친숙도가 낮을 때, 마케팅 커뮤니케이션을 통해 구매 의도나 브랜드 인지와 같은 속성들 보다 먼저 형성될 것으로 판단된다. 또한 제품의 속 성들에 대한 정보보다는 시각적 이미지와 컬러 로 인해 형성되는 태도를 측정하고자 하는 것 이기 때문에 브랜드 태도가 본 연구의 주장을 뒷받침할 수 있는 적절한 변수일 것으로 판단 된다.

\section{III. 연구방법}

\section{1 연구 설계}

본 연구의 실험은 브랜드 친숙도가 낮은 브 랜드, 실험대상이 경험하지 못한 브랜드로 진 행해야 하기 때문에, 국내에 진출하지 않은 〈METHOD〉라는 Total Cleaning 제품 브랜드를 선정하였고, 브랜드를 알고 있는 지에 대한 조 작검증 문항으로 모든 응답자들은 브랜드를 모 르는 것으로 확인되었다. 제품의 선택은 소비할 때, 속성에 대한 고려보다는 소비자의 정서적 각성(Emotional Arousal), 환상(Fantasy), 다감 각적 이미지(Multisensory Image)를 불러일으 키는(Hirschman and Holbrook 1982) SensorySocial 혹은 쾌락적 제품(Hedonic-Product)이고, 남성/여성, 소득, 연령 등에 따라 결과의 편차가 크지 않을 수 있는 제품으로 바디케어(body-care) 제품을 선택하였다.

브랜드 표현의 범위는 컬러를 조절할 수 있는 시각적 요인이 두드러지는 매체로 사인(sign), 로고(logo), 제품, 신문광고, 잡지광고, $\mathrm{POP}$ 광고, 웹사이트, 매장 외관으로 제한하였다.

일관성 있는 커뮤니케이션 실험에서는 실제 브랜드의 로고색인 연두색, Light Green(C66. $\mathrm{M} 0 . \mathrm{Y} 100 . \mathrm{K} 0)$ 과 연한 회색 Light $\mathrm{Gray}(\mathrm{C} 34$. M27.Y26.K0), 흰색 White(C0.M0.Y0.K0)컬러만 을 모든 매체에 주된 색으로 사용하였다.

불일치되는 커뮤니케이션 실험에서는 브랜드 의 로고색인 Light Green(C66.M0.Y100.K0), Light Gray(C34.M27.Y26.K0), White(C0.M0.Y0. $\mathrm{K} 0$ ) 컬러가, 다음에 노출되는 사인(Sign) 이미 
지 자극물에서는 짙은 갈색 계열 컬러와 밝은 분홍색인 Sepia(C55.M43.Y63.K19), Light-Pink (C2.M8.Y7.K0)컬러를 사용하여 다른 이미지를 느낄 수 있도록 하였고, 다음으로 매장 외관의 자극물에서는 다시 사인이 선명한 파란색 Cyan (C100.M20.Y24.K0) 과 밝은 노란색인 Light-Yellow (C7.M0.Y100.Y0)컬러가 되고, 매장 윈도우 디 스플레이의 컬러가 녹색 컬러 Green(C94.M0. $\mathrm{Y} 100 . \mathrm{K} 0)$ 가 사용되는 방식으로 불일치 되도록 하였다. 하나의 매체에 사용되는 컬러도 불일치 되게 컬러를 사용하였는데, 잡지광고 자극물에 사용된 제품 이미지의 컬러를 선명하고 진한 빨간색인 Madder-Red(C16.M100.Y100.K8)를 사 용하고 배경색은 차분하고 가라앉은 이미지 의 회색빛이 도는 녹색, Light Bottle-Green (C60.M26.Y55.K4) 과 밝은 회색 Light-Grey(C36. $\mathrm{M} 28 . \mathrm{Y} 28 . \mathrm{K} 0$ )를 사용하여 컬러에서 느껴지는 이미지가 상반되도록 하였다.

브랜드 컬러에서 명도(value)의 변화는 브랜
드 태도에 영향을 줄 수 있다는 선행연구(Gorn et al. 1997)에 따라 색상(hue)과 채도(chroma) 만 조절하였으며, 매체 간 컬러의 불일치를 위 해 컬러의 선택은 '컬러이미지스케일'에서(〈그 림 1> 참조) 이미지가 다른(멀리 떨어진) 컬러 (김진한 2002)를 사용 하였다. 컬러 이외의 다 른 시각적 요인에 있어 각 디자인의 모양, 사진, 서체, 위치, 레이아웃을 각 자극물과 자극물 안 의 각각 매체의 사용에 모두 같은 디자인 요소 가 사용되었으며, 통일 되었다. 각 매체의 표현 물은 로고, 사인(sign), 매장외관, 제품, 웹사이 트, 신문광고, 잡지광고, 배너광고, $\mathrm{POP}$ 광고의 순으로 하나씩 보여 졌으며, 자극물의 각 매체 사이에는 filler로 브랜드와 상관없는 이미지들 이 삽입되었다. 각 실험군 모두 동일한 장소와 시간에, 한 명당 하나의 컴퓨터 화면으로 노출 되었고, 일관성과 불일치 자극물이 다른 실험군 에 노출되지 않도록 하였다. 자극물이 노출되는 시간은 각 개인이 조절할 수 있도록 하였으나,

\section{〈그림 1〉컬러 이미지 스케일}
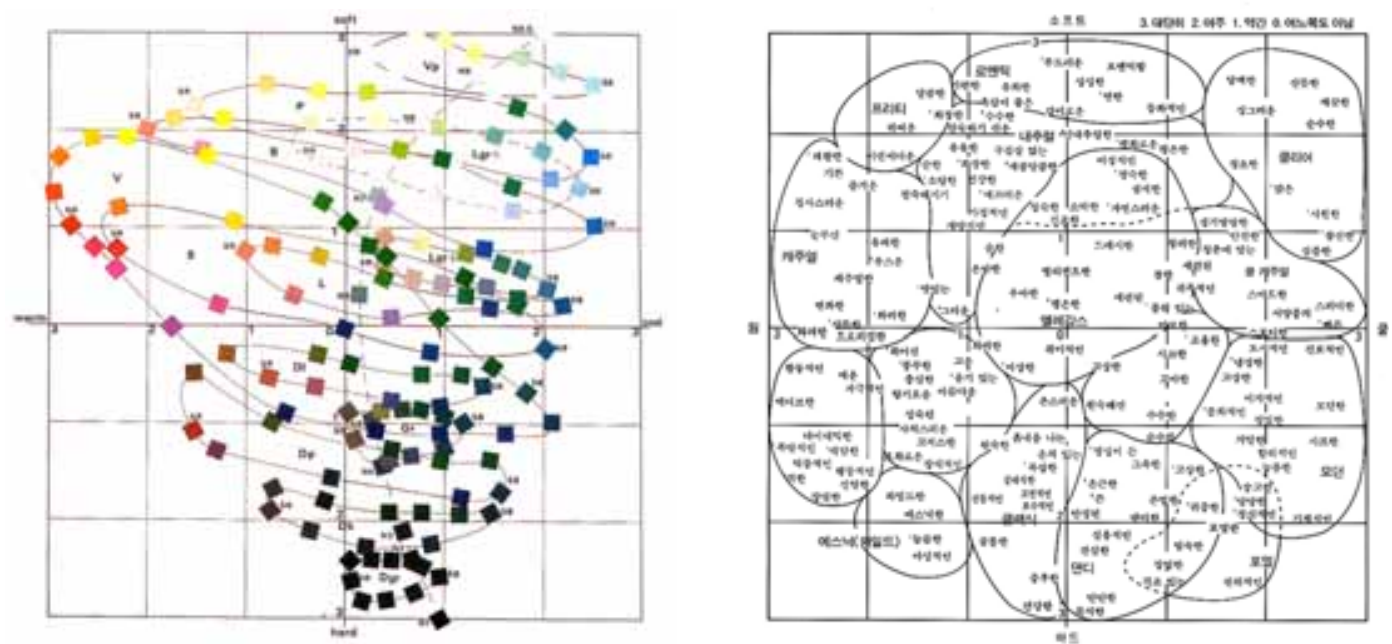
한번 만 보도록 제어하였다. 모든 문항은 자극 물을 본 후에 응답되었다.

실험은 서울소재 4년제 C대학교 학생 61명을 대상으로 2008년 11월에 실행되었다. 61개의 응 답 중 불성실한 응답 1 개를 제외한 60 개의 응 답을 분석에 사용하였다. 응답자의 구성은 남성 이 $25 \%$ (15명), 여성이 $75 \%$ (45명)를 차지하였으 며, 연령은 24세 이하가 $88.4 \%$ (53명)로 20대 초 반에 집중되었다.

\section{2 변수의 조작적 정의 및 측정}

\subsection{1 종속변수: 브랜드태도}

브랜드 태도는 브랜드 혹은 제품에 대한 측정 대상의 전체적 평가이다. 많은 연구에서 여러 가지 유사한 척도가 사용되었는데, 본 연구에서 는 여러 연구에서 사용된 척도를 바탕으로 양 극형용사(bipolar adjective)를 통한 브랜드 태도 를 측정하였다.

사용된 척도는 '싫다/좋다(J.Aaker and Maheswaran 1997)', '부정적인/긍정적인(Sujan and Bettman 1989)', '나쁜/좋은(J.Aaker and Maheswaran 1997; Burnkrant and Unnava 1995; Rossiter and Percy 1980; Sujan and Bettman 1989)', '지루한/재미있는(Rossiter and Percy 1980)', '불 쾌한/기분좋은(Burnkrant and Unnava 1995; Rossiter and Percy 1980)', '비판적인/호의적인', '비호감의/호감가는(Sujan and Bettman 1989)', '매력없는/매력적인(Babin and Burns 1997)', '비 싸지 않은/비싼(Peterson, Wilson, and Brown 1992)', '품질이 나쁜/품질이 좋은(Peterson, Wilson, and Brown 1992)', '특별한/평범한(Peterson,
Wilson, and Brown 1992)' 으로 11개의 항목을 사용하였다.

\subsection{2 조절변수: 브랜드와 소비자 자아와의 일치}

본 연구는 브랜드 컬러의 불일치가 브랜드 태 도에 미치는 영향에 조절적 영향을 미치는 요 소로 브랜드-소비자 자아와의 일치를 가정하였 으며, 브랜드-소비자 자아와의 일치는 소비자가 특정한 상황에서 자신의 자아이미지(self-image) 와 일치되는 특정한 어떤 행동에 대한 정도 (Sirgy et al. 1997)라 할 수 있다. 리커트척도 (Likert-type), 7점 척도를 사용하였으며, Sirgy 외(1997) 연구에서 사용된 5개의 측정항목 중 4 개의 항목- '이 브랜드는 나를 반영하는 것 같 다, '나와 비슷한 사람들이 이 브랜드를 사용할 것 같다', '이 브랜드는 나와 닮은 것 같다', '이 브랜드는 나의 이미지를 투영하는 것 같다'를 사용하여 측정하였다.

\subsection{3 조작검증}

1)실험에 사용된 브랜드 〈METHOD〉가 실제 로 친숙하지 않은(unfamiliar) 브랜드임을 확인 하기 위해 브랜드를 알고 있는지, 본 적이 있는 지에 대한 항목을 측정하였다. 2)실험에 사용된 바디제품에 대한 경험, 익숙한 정도에 따른 결과를 측정하기 위해 Beatty와 Smith(1987), Beatty와 Talpade(1994)의 제품에 대한 경험도 3 개의 항목을 측정하였다. 3)컬러의 일관성/불 일치에 대한 조작검증을 위해 본 브랜드에 사 용된 각 매체의 컬러 평가를 일관성, 통일성, 
조화성에 대해 3 개의 항목을 사용하였다. 제품 에 대한 경험도와 일관성 항목은 리커트척도 (Likert-type), 7점 척도를 사용하였다.

\section{IV. 연구 가설 검정}

\section{1 신뢰도 분석 및 조작검증}

\subsection{1 신뢰도 분석}

연구모델에서 제시하고 있는 가설을 검증하기 에 앞서 각 측정 변수들의 신뢰성 검증을 실행 하였다. 브랜드 태도와 소비자 자아일치 항목을 검증하였으며, 분석은 Cronbach' Alpha 계수를 이용하여 분석하였다. 신뢰도는 브랜드 태도 항 목은 .861, 소비자 자아일치 항목은 .917로 모두 .7 이상으로 신뢰성이 있다고 판단되어 항목을 이후 분석에 사용하였다.

\subsection{2 조작검증}

실험에 사용한 브랜드에 대한 친숙도를 평가 한 결과 모든 응답자가 실험에 사용된 브랜드 를 모르는 것으로 나타났으며, 제품에 대한 경 험도는 결과에 미치는 영향이 없는 것으로 나 타났다. 실험에 사용된 자극물의 각 매체 간 컬 러의 조작이 잘 이루어졌는지 점검하기 위한 일관성을 평가하였으며, '일관된 자극물'과 '불 일치된 자극물'의 각 실험 대상 그룹에 대한 t-test 분석 결과가 평균 일관(.362), 불일치(-.233), 유의확률 .023으로 나타나 자극물의 조작이 잘
이루어졌다고 볼 수 있다.

연구모형에서 제시하고 있는 각 구성개념들의 타당성을 분석하기 위해 탐색적 요인분석과 확 인적 요인분석을 실행하였다. 주성분 분석을 통 한 직각회전(varimax)법을 이용하였으며, 결과 는 〈표 2〉와 같다. 브랜드태도 항목 중 '특별 한/평범한항목은 제거되었다.

〈표 2〉 요인분석 결과

\begin{tabular}{|c|c|c|c|c|}
\hline & 항목 & $\begin{array}{l}\text { Factor } \\
\text { loading }\end{array}$ & 고유값 & $\%$ 분산 \\
\hline \multirow{6}{*}{$\begin{array}{c}\text { 전체적 } \\
\text { 브랜드 } \\
\text { 태도 }\end{array}$} & 1 & .848 & \multirow{6}{*}{3.795} & \multirow{6}{*}{18.974} \\
\hline & 2 & .799 & & \\
\hline & 3 & .736 & & \\
\hline & 4 & .665 & & \\
\hline & 5 & .658 & & \\
\hline & 6 & .616 & & \\
\hline \multirow{4}{*}{$\begin{array}{l}\text { 자아 } \\
\text { 일치성 }\end{array}$} & 1 & .918 & \multirow{4}{*}{3.426} & \multirow{4}{*}{17.132} \\
\hline & 2 & .875 & & \\
\hline & 3 & .874 & & \\
\hline & 4 & .757 & & \\
\hline \multirow{4}{*}{$\begin{array}{c}\text { 브랜드 } \\
\text { 태도 } \\
\text { (지각된 품질, } \\
\text { 감정) }\end{array}$} & 1 & .815 & \multirow{4}{*}{2.910} & \multirow{4}{*}{14.548} \\
\hline & 2 & .799 & & \\
\hline & 3 & .648 & & \\
\hline & 4 & .567 & & \\
\hline \multirow{3}{*}{$\begin{array}{c}\text { 컬러조작 } \\
\text { 평가 }\end{array}$} & 1 & .941 & \multirow{3}{*}{2.407} & \multirow{3}{*}{12.034} \\
\hline & 2 & .912 & & \\
\hline & 3 & .743 & & \\
\hline \multirow{3}{*}{$\begin{array}{c}\text { 제품에 대한 } \\
\text { 경험 }\end{array}$} & 1 & .911 & \multirow{3}{*}{2.210} & \multirow{3}{*}{11.051} \\
\hline & 2 & .762 & & \\
\hline & 3 & .733 & & \\
\hline
\end{tabular}


〈표 3〉일변량 분산분석의 기술통계량 결과

\begin{tabular}{cccc}
\hline 자아일치성 & 컬러조작 & 평균 & 표준편차 \\
\hline 자아일치성 낮음 & 일관성 & -.7180 & .62705 \\
& 불일치 & .4456 & .88171 \\
& 합계 & -.1578 & .95205 \\
\hline 자아일치성 높음 & 일관성 & .0914 & 1.21645 \\
& 불일치 & .1865 & .84134 \\
& 합계 & .1374 & 1.03564 \\
\hline 합계 & 일관성 & -.2863 & 1.05373 \\
& 불일치 & .3068 & .85432 \\
& 합계 & .0000 & 1.00000 \\
\hline
\end{tabular}

종속변수: 전체적 브랜드 태도

〈표 4〉일변량 분산분석의 개체-간 효과 검정

\begin{tabular}{ccccc}
\hline 소스 & 자유도 & 평균제곱 & $\mathrm{F}$ & 유의확률 \\
\hline 수정 모형 & 3 & 3.484 & 4.042 & .012 \\
절편 & 1 & .000 & .000 & .991 \\
자아 일치성 & 1 & 1.091 & 1.266 & .265 \\
컬러조작 & 1 & 5.708 & 6.622 & .013 \\
자아 일치성 * 컬러조작 & 1 & 4.114 & 4.773 & .033 \\
오차 & 54 & .862 & & \\
합계 & 58 & & & \\
수정 합계 & 57 & & & \\
\hline
\end{tabular}

종속변수: 전체적 브랜드 태도

\section{2 가설검정}

가설 1 은 매체 간 불일치에 따른 브랜드 태도 는 매체 간 일관성 보다 긍정적일 것이며, 가설 2 는 브랜드의 불일치가 브랜드 태도에 미치는 영향에 자아일치성이 조절효과를 가진다는 것 이다. 이를 검증하기 위해 일변량 분산분석을 실시하였으며, 그 결과는〈표 3〉과 같다.

모형에 대한 결과(〈표 4〉 참고)는 $\mathrm{F}(3,57)=$
4.042, $\mathrm{p}=.012$ 로 연구에서 설정한 모형이 적합하 며, 컬러조작에 대한 유의한 주효과 $(p=0.013)$ 가 나타나, 가설 1 이 지지됨을 알 수 있다.

컬러의 불일치가 브랜드 태도에 미치는 영향 에 자아일치성의 조절효과에 대한 결과는 $\mathrm{F}(1$, $57)=4.773, \mathrm{p}=.033$ 으로 유의한 결과가 나타났다. 또한 그 평균이 자아일치성이 낮은 경우, 일관 성 평균 -.7180 , 불일치 평균 .4456 이고, 자아일 치성이 높은 경우, 일관성 평균 .0914, 불일치 
평균 .1865으로 조절 효과의 정도가 자아일치성 이 낮은 경우에 더 크게 나타나 가설2가 지지 되었다.

\section{〈그림 2〉 전체적 브랜드 태도의 추정된 주변 평균}

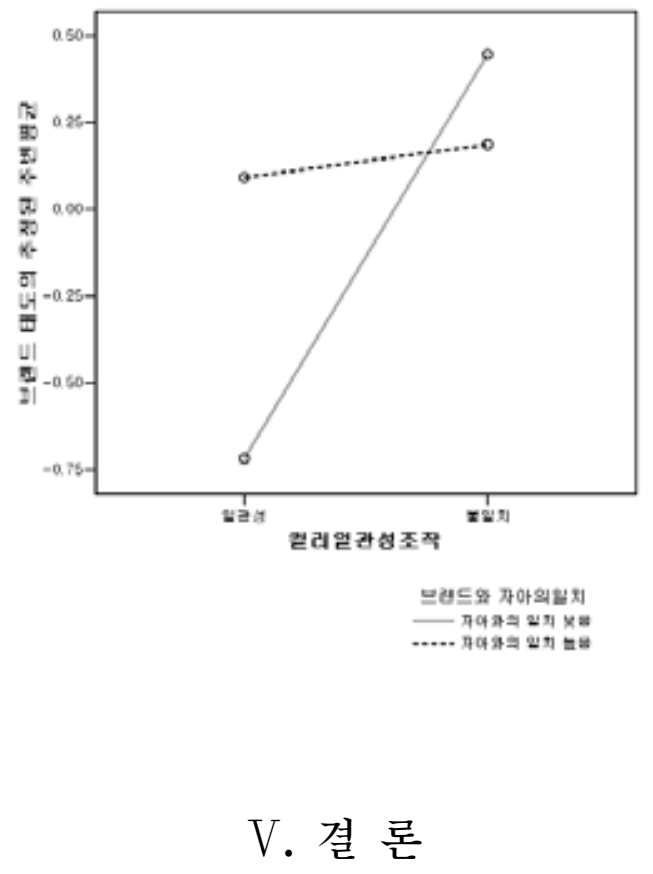

\section{1 연구결과 요약 및 시사점}

본 연구는 통합적 브랜드 전략으로 브랜드 접 점, 매체 간의 컬러 불일치가 브랜드 태도에 미 치는 영향과 자아 일치성의 조절효과를 중심으 로 연구를 진행하였다. 연구의 가설은 브랜드 친숙도가 낮은 브랜드의 경우, 매체 간 컬러의 불일치는 컬러가 일치하는 브랜드 전략에 비해 브랜드 태도에 긍정적 영향을 미친다는 것과 그 영향에 자아 일치성이 조절적 영향이 미치 며 그 정도는 자아일치가 낮은 경우에 더 크다
는 것이다. 본 연구는 이를 검증하기 위해 실험 을 통한 분석을 한 결과 매체 간 컬러의 불일 치에 따른 브랜드 태도는 컬러의 일관성에 따 른 브랜드 태도에 비해 높게 나타나 가설 1이 지지되었다. 소비자의 자아일치성에 따른 조절 효과에 대한 결과는 자아일치가 낮은 경우에 높은 경우보다 태도의 변화가 양의 방향으로 높게 나타나 가설2도 지지되었다.

본 연구는 브랜드를 전달하는 전략 중 시각적 인 요소를 활용하여 브랜드에 대해 긍정적인 효과를 가져 올 수 있는 방법으로 컬러를 제안 하였다. 일반적으로 브랜드 관리에 있어 매체 간 시각적 요인은 컬러를 비롯하여 모든 시각 적 요인을 일관되게 사용하고 매체에 특성에 맞게 맞추는 정도의 변화만이 허용되고 있다. 언어적 요소 뿐 아니라 시각적 요소가 일관되 게 유지되어야만 소비자에게 어필할 수 있다는 것이다.

그러나 본 연구는 시각적 요인의 적절한 불일 치는 오히려 소비자, 특히 브랜드에 대해 동화 (elaboration) 되지 않은 소비자의 브랜드 태도를 높일 수 있다는 결과를 도출하고 브랜드의 메 시지를 일관성 있게, 혹은 명확하게 전달하면서 소비자의 태도를 높일 수 있는 적절한 불일치 를 활용하는 방법으로써 컬러가 효과적인 도구 가 될 수 있다는 가능성을 증명함으로써 브랜 드 관리의 새로운 전략을 제시하였다.

본 연구의 매체 간 불일치는 한 브랜드에 대 해 여러 매체에서 느껴지는 느낌, 즉 브랜드 이 미지가 다르다는 것을 의미한다. 다르게 말하면, ‘의외성'이 있다는 것이며, 의외성은 많은 정보 중에서 무의식적으로 지나칠 수 있는 정보에 관심을 기울일 수 있는 가능성을 높여준다는 
것이다.

이 전략은 브랜드 친숙도가 낮은 소비자, 즉 브랜드를 모르는 소비자에게 긍정적인 태도를 불러왔으며, 그 중 브랜드-소비자의 자아가 일 치하지 않는 그룹에게 더욱 긍정적인 태도를 가져왔다는 데에 시사점이 있다고 할 수 있다.

브랜드가 궁극적으로 전달하고자하는 메시지 가 변하지 않는 범위 내- 수락범위 내에서의 불일치는 브랜드 인지도를 높이고, 브랜드 태도 를 형성하는 데에 긍정적인 역할을 할 수 있을 것이다. 소비자 특성 측면에서는 타겟 소비자 뿐 아니라, 브랜드가 속한 카테고리에 관심을 가질 가능성이 낮은 그룹, 잠재 타겟까지 그 영 향을 미칠 수 있어 브랜드 도입기의 커뮤니케 이션 전략으로 사용될 때 이전의 '일관적 표현' 를 통한 방법보다 더욱 효율적일 것이다.

본 연구에서 제안한 매체 간 컬러의 불일치는 같은 시기에 매체 별로 컬러를 불일치하는 것 으로, 많은 브랜드들이 사용하는 특정 시기에 따라 브랜드의 모든 컬러를 변화시키는 전략과 는 구분되어야 할 것이다. 소비자는 한 매체를 통해 형성한 스키마에 따라 다른 매체에서 불 일치하였을 때 그 브랜드에 대해 이해하려는 노력을 하고, 브랜드 태도를 형성하는 것이다.

본 연구에서는 적절한 불일치의 한 방법으로 컬러를 불일치시키는 방법을 사용하였다. 컬러 의 사용에 있어 명도(value)의 변화는 브랜드 태도에 영향을 줄 수 있다는 선행연구(Gorn et al. 1997)에 따라 색상(hue)과 채도(chroma)만 변경하고, 색상은 '컬러 이미지 스케일'을 참고 하여 이미지가 다른 색을 사용하였다. 컬러의 사용 시 근접색을 사용하는 것은 소비자의 지 각을 자극시킬 수 없음을 인지하고 컬러의 사
용에 유의하여야 할 것이다.

본 연구의 결과는 일반적으로 실행되던 브랜 드 관리 전략의 일관성 분야를 적절한 불일치 로써 성숙브랜드(mature brand)를 리포지셔닝 (repositioning)하는 목적 이외에도 신생 브랜드 의 인지를 높이고 태도를 형성시키는 전략으로 범위를 확장시켰으며, 브랜드 표현의 다양성 수 준을 실증적으로 보여주었다는 면에서 의의가 있다고 할 수 있다.

\section{2 연구의 한계점 및 향후 연구}

본 연구는 다음과 같은 한계점이 존재한다. 첫 째, 본 연구는 제품을 비교적 컬러의 사용이 자 유로운 바디제품을 사용하여 실증하였다. 그러 나 브랜드나 제품, 혹은 문화에 따라 그 영향이 달라질 수 있을 것으로 생각된다. 특히 컬러에 민감한 제품인 식품과 같은 제품에서는 영향이 달라질 수 있을 것이다. 카테고리, 문화별로 그 영향을 살펴보아야 할 것이다. 둘째, 본 연구는 브랜드에 대한 지식이 없는 친숙도가 낮은 브랜 드를 대상으로 하였다. 그러나 이미 브랜드에 대한 컬러의 인식이 확고한 브랜드에 대한 영향 이나 컬러 이외에 이미 소비자가 인식하고 있는 스키마가 명확한 브랜드에는 그 영향이 달라질 것이다. 셋째, 본 연구는 불일치에 따른 효과로 브랜드 태도를 보았으나, 문헌 연구에서 언급하 였던 브랜드 회상에 대한 효과를 보지 못한 것 이 그 한계라 할 수 있다. 불일치라는 자극에 의한 영향이기 때문에 회상에 있어서도 장기적 인지 단기적인지 또한 보아야 할 것이다. 마지 막으로 본 연구에서 컬러의 불일치를 메시지를 일관적으로 가져가면서 소비자의 태도를 높일 
수 있는 '적절한 불일치'로 제시하였다. 향후 연 구에서는 컬러 뿐 아니라 다른 시각적 요소에 대한 영향을 살펴보아야 할 것이며, 완전한 불 일치'에 대한 연구를 통해 불일치 수준에 따른 영향을 보아야 할 것이다.

〈논문 접수일: 2009. 08. 10〉

〈게재 확정일: 2009. 12. 04〉

\section{참고문헌}

김일철(역)(2003), 브랜드 커뮤니케이션(Duncan,

Tom and Sandra Moriarty, Driving Brand

Value), 서울: 북코리아

김진한(2002), 색채의 원리, 서울: 시공사

배상철(1998), “스플릿 TV 광고의 인지적 효과

에 관한 연구," 광고연구, 41, 141-168

최원주, 김흥규(2005), 브랜드는 커뮤니케이션이

다, 서울: 커뮤니케이션북스

Aaker, David A.(1996), Building Strong Brands, NY: The Free Press

Aaker, Jennifer L. and Durairaj Maheswaran (1997), "The effect of cultural orientation on persuasion," The Journal of Consumer Research, 24(3), 315-328

Aaker, Jennifer L., Susan Fournier, and S. Adam Brasel(2004), "When good brands do bad," The Journal of Consumer Research, $31(1), 1-16$

Babin, Laurie and Alvin C. Burns(1997), "Effect of print Ad pictures and copy containing instructions to imagine on mental imagery that mediates attitudes," Journal of Advertising, 26(3), 33-44

Beatty, Sharon E. and Scott M. Smith(1987), "External search effort: an investigation across several product categories," The Journal of Consumer Research, 14(1), 8395

Bellizzi, J., A. E. Crowley, and R. W. Hasty (1983), "The effects of color in store design," Journal of Retailing, 59(1), 21-45

Berger, Ida E., and Andrew A. Mitchell(1989), "The effect of advertising on attitude accessibility," The Journal of Consumer Research, 16(3), 280-288

Berlyne, D. E.(1971), Aesthetics and Psychobiology, NY; Eton Century Crofts, cited by Gianluigi Guido(2001), The Salience of Marketing Stimuli: An Incongruity-salience Hypothesis on Consumer Awareness, Kluwer Academic Publishers Group

Bhattacharya, C. B. and Sankar Sen(2003), "Consumer-Company identification: a framework for understanding consumers' relationships with companies," The Journal of Marketing, 67(2), 76-88

Birdwell, AL E.(1968), "A study of the influence of image congruence on consumer choice," Journal of Business, 41(1), 76-88

Bornstein, Marc H.(1989), "Sensitive periods in development: structural characteristics and causal interpretations," Psychological Bulletin, 105(2), 179-197

Broniarczyk, Susan M. and Joseph W. Alba 
(1994), "The importance of the brand in brand extension," Journal of Marketing Research, 31(2), 214-228

Bottomley, Paul A. and John R. Doyle(2006), "The interactive effects of colors and products on perceptions of brand logo appropriateness," Marketing Theory, 6(1), 63-83 Bruce, V. and P.R. Green(1990), Visual perception: Physiology, Psychology, and Ecology, Hove, UK: Lawrence Erlbaum

Burnkrant, Robert E. and H. Rao Unnava (1995), "Effects of self-referencing on persuasion," The Journal of Consumer Research, 22(1), 17-26

Campbell, Margaret C. and Ronald C. Goodstein (2001), "The moderating effect of perceived risk on consumers' evaluations of product incongruity: preference for the norm," The Journal of Consumer Research, 28(3), 439449

Chattopadhyay, Amitava and Joseph W. Alba (1988), "The situational importance of $\mathrm{re}^{-}$ call and interface in consumer decision making," The Journal of Consumer Research, 15(1), 1-12

Dolich, Ira J.(1969), "Congruence relationships between self images and product brands," Journal of Marketing Research, 6(1), 8084

Fabiani, M., and E. Donchin(1995), "Encoding processes and memory organization: a model of the von Restorff effect," Journal of Experimental Psychology, Learning, Memory, and Cognition, 21(1), 224-240

Fournier, Susan(1998), "Consumers and their brands: developing relationship theory in consumer research," The Journal of Consumer Research, 24(4), 343-373.

Garber, Lawrence L., and Eva M. Hyatt(2003), "Color as a tool for visual persuasion," in Linda M. Scott and Rajeev Batra, Persuasive imagery: a consumer response perspective, Lawrence Erlbaum Associates Publishers

Gorn, Gerald J., Amitava Chattopadhyay, Tracey Yi, and Darren W. Dahl(1997), "Effects of color as an executional cue in advertising: They're in the shade," Management Science, 43(10), 1387-1400

Graeff, Timothy R.(1996), ”Image congruence effects on product evaluations: the role of self-monitoring and public/private consumption," Psychology and Marketing, 13(5) 481-500

Guido, Gianluigi(2001), The Salience of Marketing Stimuli: an incongruity-salience hypothesis on consumer awareness, Kluwer Academic Publishers Group

Heckler, Susan E. and Terry L. Childers(1992), "The role of expectancy and relevancy in memory for verbal and visual information: what is incongruency?," The Journal of Consumer Research, 18(4), 475-492

Hirschman, Elizabeth C. and Morris B. Holbrook (1982), "Hedonic consumption: emerging concepts, method and propositions," The 
Journal of Marketing, 46(3), 92-101

Keller, Kevin Lane(1993), "Conceptualizing, measuring, and managing customer-based brand equity," The Journal of Marketing, 57(1), 1-22

Keller, Kevin Lane(1998), Building, measuring, and managing brand equity, NJ: Prentice Hall

Keller, Kevin Lane(2003), "Brand synthesis: the multidimensionality of brand knowledge," The Journal of Consumer Research, 29(4), 595-600

Kent, Robert J. and Chris T. Allen(1994), "Competitive interference effects in consumer memory for advertising: the role of brand familiarity," The Journal of Marketing, 58(3), 97-105

Kirmani, Amna and Baba Shiv(1998), "Effects of source congruity on brand attitudes and beliefs: the moderating role of issuerelevant elaboration," Journal of Consumer Psychology, 7(1), 25-47

Kishiyama, Mark M. and Andrew P. Yonelinas (2003), "Novelty effects on recollection and familiarity in recognition memory," Memory and Cognition, 31(7), 1045-1051

Kressmann, Frank, M. Joseph Sirgy, Andreas Herrmann, Frank Huber, Stephanie Huber and Dong-Jin Lee(2006), "Direct and indirect effects of self-image congruence on brand loyalty," Journal of Business Research, 59(9), 955-964

Lange, Fredric and Micael Dahlen(2003), "Let's be strange: brand familiarity and ad-brand incongruency," Journal of Product and Brand Mangement, 12(7), 449-461

Lee, S. and J. H. Barnes, Jr.(1990), "Using color preferences in magazine advertising," Journal of Advertising Research, 29(6), 25-30

Lee, Yih Hwai and Charlotte Mason(1999), "Responses to information incongruency in advertising: the role of expectancy, relevancy, and humor," The Journal of Consumer Research, 26(2), 156-169

Lee, Yih Hwai(2000), "Manipulating ad message involvement through information expectancy: effects on attitude evaluation and confidence," Journal of Advertising, 29 (2), $29-43$

Leigh, James H.(1992), "Modality congruence, multiple resource theory and intermedia broadcast comparisons: an elaboration," Journal of Advertising, 21(2), 55-62

Light, Larry(1994), Bringing research to the brand equity process, ARF brand equity workshop, February 15-16, Cited by Keller, Kevin Lane (1998), Building, measuring, and managing brand equity, NJ: Prentice Hall

Machleit, Karen A., Chris T. Allen, and Thomas J. Madden(1993), "The mature brand and brand interest: an alternative consequence of ad-evoked affect," The Journal of Marketing, 57, 72-82

Mandler, George(1982), “The structure of value: 
accounting for taste," in Clark, Margaret S. and ST Fiske(eds), Affect and cognition, NJ: Lawrence Erlbaum Associate, 3-36

Malhotra, Naresh K.(1988), "Self-concept and product choice: an integrated perspective," Journal of Economic Psychology, 9(1), 128

McQuarrie, Edward F. and David Glen Mick (1999), "Visual rhetoric in advertising: textinterpretive, experimental, and reader-response analyses," The Journal of Consumer Research, 26(1), 37-54

Meyers-Levy, Joan and Alice M. Tybout(1989), "Schema congruity as a basis for product evaluation," The Journal of Consumer Research, 16(1), 39-54

Meyers-Levy, Joan. and Laura A. Peracchio (1995), "Understanding the effects of color: how the correspondence between available and required resources effects attitudes," The Journal of Consumer Research, 22(2), 121-138

Moore, Jeri, and Esther Thorson(1996), "Strategic planning for integrated marketing communications programs: an approach to moving from chaotic toward systematic," in Esther Thorson, Jeri Moore(1996), Integrated Communication: synergy of persuasive voices, N.J; Erlbaum, 135-152

Muehling, Darrel D. and Russell N. Laczniak (1988), “Advertising's immediate and delayed influence on brand attitudes: considerations across message-involvement levels," Journal of Advertising, 17(4), 23-34

Park, C. Whan, Bernard J. Jaworski, and Deborah J. Maclnnis(1986), "Strategic brand concept-image management," The Journal of Marketing, 50(4), 135-145

Peterson, Robert A., William R. Wilson, and Steven P. Brown(1992), "Effect of advertised customer satisfaction claim on consumer attitudes and purchase intentions," Journal of Advertising Research, 32(2), $34-40$

Peracchio, Laura A. and Alice M. Tybout (1996), "The moderating role of prior knowledge in schema-based product evaluation," The Journal of Consumer Research, 23(3), 177-192

Petty, Richard E. and John T. Cacioppo (1981), Attitudes and persuasion: classic and contemporary approaches, IA: William C. Brown

Pimentel, Ronald W. and Susan E. Helker (2003), "Changes in logo design: chasing the elusive butterfly curve," in Linda M Scott and Rajeev Batra(ed.), Persuasive imagery: a consumer response perspective, Lawrence Erlbaum Associates Publishers

Rossiter, John R. and Larry Percy(1980) "Attitude change through visual imagery in advertising," Journal of Advertising, 9(2), 10-16

Rossiter John R. and Larry Percy(1987), "Advertising and Promotion Management," McGraw-Hill 
Schmitt, Bernd H., Alex Simonson(1997), Marketing aesthetics: the strategic management of brands, identity and image, Free Press

Schindler, P. S.(1986), "Color and contrast in magazine advertising," Psychology and Marketing, 3(2), 69-78

Scott, Linda M. and Rajeev Batra(2003), Persuasive Imagery: a consumer response perspective, Lawrence Erlbaum Associates Publishers

Shimp, Terence A.(1999), Advertising, promotion, and supplemental aspects of integrated marketing communications, South-Western, Div of Thomson Learning(5th ed)

Sirgy M. Joseph, Samli CA.(1985), "A path analytic model of store loyalty involving self-concept, store image, socioeconomic status, and geographic loyalty," Journal of the Academy of Marketing Science, 13(3), 265- 291

Sirgy M. Joseph.(1985), "Using self-congruity and ideal congruity to predict purchase motivation," Journal of Business Research, 13(3), 195-206

Sirgy, M. Joseph(1997), Integrated Marketing Communications: a systems approach, Prentice Hall College Div(1st ed)

Sirgy, M. Joseph, Dhruv Grewal, Tamara F. Mangleburg, Jea-Ok Park, Kye-Sung Chon, C.B. Claiborne, J.S. Johar, and Harold Berkman(1997), "Assessing the predictive validity of two method of measuring self- image congruence, Journal of the Academy of Marketing Science," 25(3), 229-241

Sheriff, M. and Hovland, C.I.(1961), Social judgment: assimilation and contrast effect in communication and attitude change, New Haven, CT: Yale University Press, cited by Ronald W. Pimentel and Susan E. Helker(2003), Changes in logo design: chasing the elusive butterfly curve, Lawrence Erlbaum Associates Publishers

Sjödin, Henrik and Fredrik Törn(2006), "When communication challenges brand associations: a framework for understanding consumer responses to brand image incongruity," Journal of Consumer Behaviour, 5(1), 3242

Smith, Peter W., Richard A. Feinberg, and David J. Burns (1998), “An examination of classical conditioning principles in an ecologically valid advertising context," Journal of Marketing Theory and Practice, 6(1), 63-72

Sparkman, R. Jr. and L. M. Austin(1980), "The effect on sales of color in newspaper advertisement," Journal of Advertising, 9 (4), 39-42

Sujan, Mita and James R. Bettman(1989), "The effects of brand positioning strategies on consumers' brand and category perceptions: some insights from schema research," Journal of Marketing Research, 26 (4), 454-467

Tavassoli, Nader T.(1998), "Language in mul- 
timedia: interaction of spoken and written information," The Journal of Consumer Research, 25(1), 26-38

Thorbjörnsen Helge, Magne Supphellen, Herbjorn Nysveen, Per Egil Pedersen(2002), "Building brand relationships online: a comparison of two interactive applications," Journal of Interactive Marketing, 16(3), 17-34

Triesman, A.(1991), "Search, similarity, and integration of features between and within dimensions," Journal of Experimental Psychology, Human perception and performance, 17(3), 652 -676

Unnava, H. Rao(1996), "Interactive effects of presentation modality and message-generated imagery on recall of advertising information," The Journal of Consumer Research, 23(1), 81-89
Wallace, W. P. (1965), "Review of the historical, empirical, and theoretical status of the von Restorff phenomenon," Psychological Bulletin, 63, 410-424

Zajonc, Robert B.(1968), "Attitutional effects of mere exposure," Journal of Personality and Social Psychology, 9(2), 1-27, cited by Scott, Linda M. and Rajeev Batra (2003), Persuasive Imagery: a consumer response perspective, Lawrence Erlbaum Associates Publishers

Zaltman, Gerald (1996), "Metaphorically speaking: new technique uses multidisciplinary ideas to improve qualitative research," Marketing research: a quarterly business management publication of the American Marketing Association, 8(2), 13-20 


\title{
The Effect of Color Incongruity on Brand Attitude: Moderating Effect of Self-Image Congruence
}

\author{
Lee, Sang Eun* \\ Kim, Sang Yong**
}

\begin{abstract}
In this research, through experiments, we show that incongruity of color between mediums has positive influence on brand attitude in terms of integrated management of brand. We also present that self-image congruence of 'brand-consumer' has moderating effect on such influence of color incongruity. Mediums were limited to the ones that magnifying visual influence in order only to observe influence of color. With the same reason, visual factors other than color were coherently set or held constant and we chose brands with either low familarity or no previous knowledge.

As a result, we find that brand attitude by the incongruity of color between mediums was higher compared to brand attitude by the congruence of color. In case with lower self-image congruence of brand-consumer we show higher change in attitude compared to the one with higher self-image congruence of brand-consumer. We believe our findings are interesting to note that brand may be enhanced by forming positive brand attitude through brand expression i.e., color of visual factors. In addition, we suggest that level of congruence and diversity of brand expression is in fact deeper or wider than that of brand manager's intuition. We see that it is possible for studying brands the incongruity which has been studied as a strategy to reposition mature brands can be a way of improving the recognition on new brands.
\end{abstract}

Key words: color incongruity, incongruity-salience, schema theory, mismatch, brand attitude, self-image congruence

\footnotetext{
* Lecturer at Woosong Univ. and Kangwon Univ. and Ph.D. student at Dept. of Visual Communication Design, Hongik University (bytaij@ hotmail.com)

** Professor, Korea University Business School(sangkim@korea.ac.kr)
} 


\section{Research Goal}

In this research, we suggest that incongruity of communication between mediums may have influence on recognition of consumer and try to find positive influence of incongruity on brand attitude. We specifically focus on effectiveness of color.

\section{Brand Congruence and Incongruity}

Research reporting congruence of brand communication tell us that maintaining congruency of visual factors such as mark and logo identifying brand is a method to increase chance to effectively approach target customers(Schmitt and Simonson 1997) and to preoccupy superiority in competition in order for customers and other interest groups to promptly and accurately recognize the brand. Concept of brand must maintain its congruence(Park et al. 1986; Keller 1993; Broniarczyk and Alba 1994; Leigh 1992; Scott and Batra 2003).

On the other hand, research reporting incongruity show us that maintaining congruence of identity may result in boringness in customer's aspect and such research on incongruity and effectiveness of relationship report that perceiver pays attention to subjects circumstantially inconsistent with one's own schema by mentioning Schema theory, Information Processing Approach, and of Incongruity-Salience. According to Guido(2001), Incongruity-Salience varies depending on circumstance of stimulus occurrence and condition of context, and that it interacts with contextual novelty and other physical properties(location, contrast, and size). According to other research supporting theory of Novelty Effect(Berlyne 1971), novelty stimulus on perceiver results influence on overall memory of recognition (Fabiani and Donchin 1995; Kishiyama and Yonelinas 2003) and it holds positive relationship to preference(Bruce and Green 1990). However, perspective on incongruity contains weakness that concept of brand cannot be delivered firmly(Schmitt and Simonson 1997, Light 1994).

\section{Moderate Incongruity}

Encoding Variability Theory of Tavassoli(1998) states that when consumer receives identical messages in diverse contexts, such massages are encoded in memory and form more stronger, definite, and approachable information to increase possibility of association. In addition, Social Judgment Theory(Sheriff and Hovland 1961) states that individual is divided into latitude of acceptance and latitude of rejection according to input message and frame of reference in 
making decision (Pimentel and Helker 2003) and it is reported that if proposed object is within perceiver's latitude of acceptance, assimilation effect occurs and it allows recognition of object to be more similar than it actually is (Scott and Batra 2003). As a result, incongruity must be within boundary of acceptance on consumer's recognition to result positive attitude. In addition, research on Schema theory on incongruity reports that consumers prefer moderate incongruity (Mandler 1982; MeyersLevy and Tybout 1989).

Moderate incongruity means that each brand factor performs respectively different role to form brand identity(Keller 1998) and that they should be managed based on particularity of each medium respectively by maintaining message with congruence rather than unilateral congruence to maximize the effectiveness(Sirgy 1997).

In this research, we define that moderate level of incongruity can be maintained by unifying all other visual and lingual cues and differentiating colors used between each medium. It is assessed that incongruity of color between each medium will result in positive influence on consumer's brand attitude in case for new brand with low familarity.

Hypothesis 1: Incongruity of color between mediums will result in positive influence on consumer's brand attitude under the condition of low familarity of brand. Said differently, if familarity of brand is low, more positive influence on consumer's brand attitude with higher level of incongruity.

\section{Self-image Congruence of Brand- Consumer}

The result of incongruity stimulus varied according to perceiver(Guido 2001). Stimulus drawing attention isn't identical for all individuals and it should interact along by combining context and individual. In incongruity theory (Guido 2001), incongruity is said to be achieved accidentally instead of exploration of information on need and it is achieved by incongruity of stimulus under condition lacking schema. In research of Kirmani \& Shiv(1998), practical result was proposed that source congruity had positive influence on brand attitude when issue-relevant elaboration level was high, whereas source congruence had low influence on brand attitude when issue-relevant elaboration level was low and that peripheral cue or heuristic method is used to form brand attitude.

Such result proposes possibility of level of attitude on factors expressing inconsistent brand between mediums can be higher on experimental subjects holding lower self-image congruence of consumer. This also suggests that it is possible not only to change attitude and recognition of consumers set as targets by new brand(with 
low brand familarity) on brand, but also other consumer's recognition and attitude on brand as well.

In this research, we propose to increase brand attitude by comparing groups of consumers holding low self-image congruence and those holding lower interest on brand.

Hypothesis 2: If brand familarity is low, positive influence level of incongruity of color between mediums on consumer's brand attitude will be higher in low self-image congruence of brand-consumer. Thus, if brand familarity is low, lower self-image congruence of brandconsumer will have higher positive influence level on consumer's brand attitude.

\section{Method}

For experiment in this research, brand with low familarity, for total cleaning, unreleased in the domestic market was selected. And we confirmed that all respondents were unfamiliar with selected brand through processing question to certify our manipulation. Selection of product was either Sensory-Social or Hedonic-Product. Body-care product was selected as product to lower deviation result based on male/female, income, and age. Boundary of brand expression was limited to mediums with significant visual factors of adjustable color such as sign, logo, product, newspaper advertisement, magazine advertisement, POP advertisement, web-site, and shop appearance.

According to previous research (Gorn et al. 1997) stating influence of value change on brand attitude in terms of brand color, only hue and chroma in color of each medium was adjusted. For incongruity of color between mediums, 'Color Image Scale(Kim, Jin Han 2002)' was used as reference and color in different image was used for selection of color. Visual factors other than color- identical design factors from respective medium in stimulus object and each stimulus object was unified and used in all shapes, pictures, fonts, locations, and layouts of each design. All questions were answered after viewing stimulus object. The respondents consisted of 25\%(15) males and 75\%(45) females. $88.4 \%$ (53) respondents were under age of 24 and age was concentrated in early 20's.

\section{Result}

As a result of evaluating familarity of brand used in experiment, it was observed that all respondents were unfamiliar with the brand used in experiment and that experience in product had no influence on result. Congruence was evaluated to inspect whether manipulation of color between respective medium of stimulus object used in experiment was processed well 
and result of t-test analysis on each subject group on experiment of 'consistent stimulus object' and 'inconsistent stimulus object', was examined as average congruence(.362), incongruity (-.233), and significance probability(.023) and it can be assessed that manipulation of stimulus objects was processed well.

One-way ANOVA was performed to prove our hypotheses. $F(3,57)=4.042, p=.012$ was achieved as a result and it was found that model selected for research was appropriate. Significant main effect $(\mathrm{p}=0.013)$ on color manipulation was observed and hypothesis 1 was supported.

For the moderate effect of self-image congruence on influence of incongruity of color on brand attitude, $\mathrm{F}(1,57)=4.773, \mathrm{p}=.033$ was observed. In addition, congruence average (-.7180) and incongruity average(.4456) were observed when self-image congruence was low and congruence average(.0914) and incongruity average (.1865) were observed when self-image congruence was high. The level of moderate effect was higher when self-image congruence was low and hypothesis 2 was supported.

\section{Research Implications}

In this research a new strategy of brand management is proposed with moderate incongruity of visual factor which can even increase consumer's brand attitude.

we find that brand attitude by the incongruity of color between mediums was higher compared to brand attitude by the congruence of color. In case with lower self-image congruence of brand-consumer we show higher change in attitude compared to the one with higher selfimage congruence of brand-consumer. We believe our findings are interesting to note that brand may be enhanced by forming positive brand attitude through brand expression i.e., color of visual factors. In addition, we suggest that level of congruence and diversity of brand expression is in fact deeper or wider than that of brand manager's intuition. We see that it is possible for studying brands the incongruity which has been studied as a strategy to reposition mature brands can be a way of improving the recognition on new brands. 